\title{
Medical Image of the Week: Fungus Ball
}

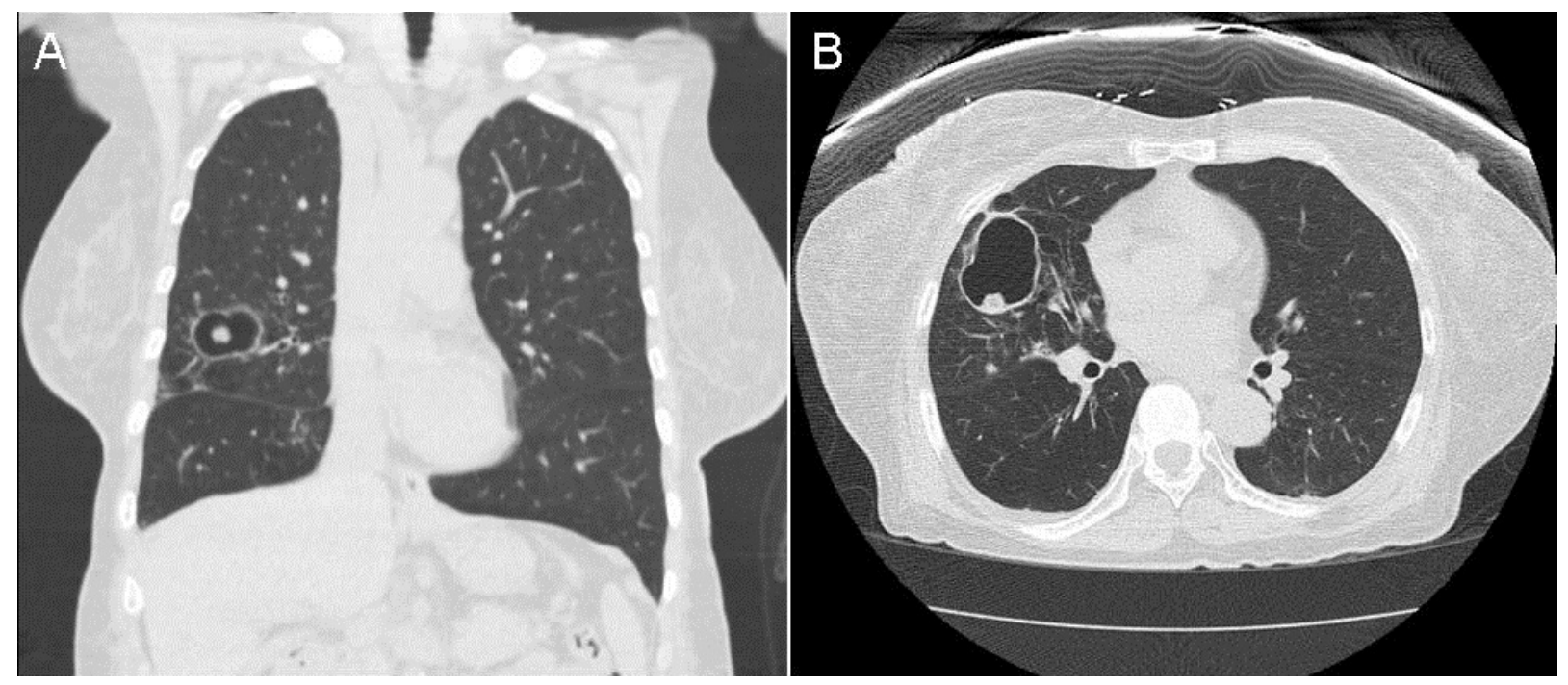

Figure 1. Panel A: Coronal view of thoracic CT scan shows a fungus ball (mycetoma) within a $4.7 \mathrm{~cm}$ thin walled cavity, Panel B: Axial image of fungus ball and thin walled cavity.

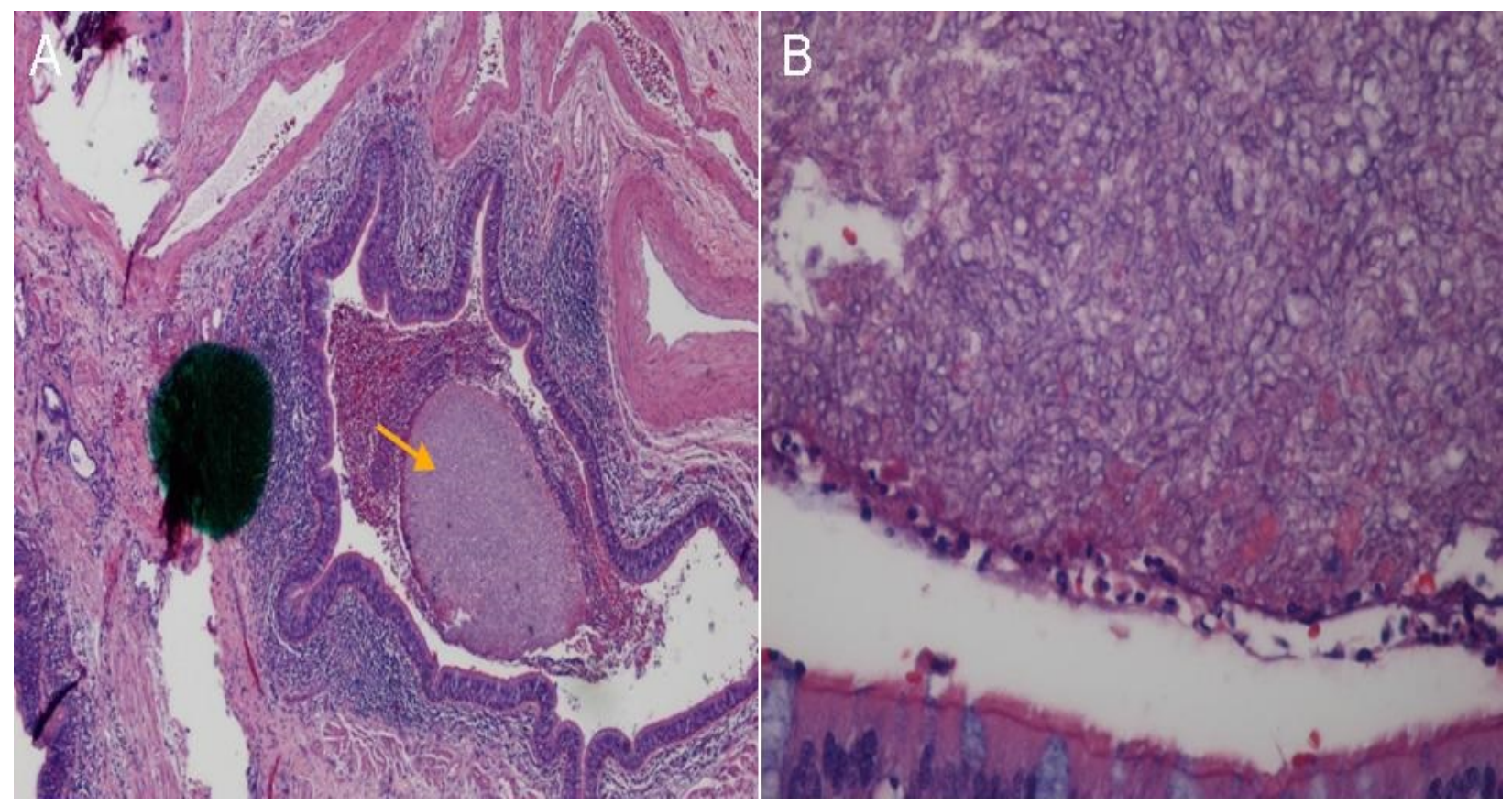

Figure 2 Panel A. Prominent eosinophilic infiltration with epithelial-lined cavity containing fungus ball (orange arrow). Panel B: Higher magnification. 
A 69 year-old Asian woman living in Arizona with a past medical history of nephrotic syndrome on high-dose steroids had worsening pulmonary symptoms. A computed tomography (CT) of the chest (Figure 1) showed a $4.7 \mathrm{~cm}$ thin walled cavitary lesion in the right middle lobe compatible with mycetoma. She underwent thoracotomy for mycetoma resection. Surgical pathology confirmed an epithelial-lined cavity containing dense mycelia (Figure 2). Given the patient lived in an endemic area; the cavity was thought to be likely due to coccidioidomycosis. However, the mycetoma was of unclear etiology. No spherules were noted on GMS stain and tissue culture was negative. While of unclear clinical significance which fungus colonizes a pre-existing cavity, a Coccidioides PCR was performed and no Coccidioides genes were amplified making a Coccidioides mycetoma very unlikely.

Pulmonary mycetoma or "fungus ball" consists of dense fungal elements and amorphous cellular material within a pre-existing pulmonary cavity. Classically presenting as an aspergilloma, other fungi can cause similar lesions. Patients with mycetoma rarely develop symptoms. When present, symptoms can include chest pain, cough, hemoptysis, fatigue, fever, or unintentional weight loss. If asymptomatic, no treatment is required. Surgical resection and/or embolization may be required in cases of severe symptoms including hemoptysis.

Scott Rosen MD, Bridget Barker PhD, Branden Larsen MD PhD, and Ishna Poojary MD Department of Medicine and Pathology University of Arizona Medical Center

Tucson, AZ and

Tgen North

Flagstaff, AZ

\section{References}

1. Winn RE, Johnson R, Galgiani JN, Butler C, Pluss J. Cavitary coccidioidomycosis with fungus ball formation. Diagnosis by fiberoptic bronchoscopy with coexistence of hyphae and spherules. Chest. 1994;105(2):412-6. [CrossRef] [PubMed]

2. Sobonya RE, Yanes J, Klotz SA. Cavitary pulmonary coccidioidomycosis: pathologic and clinical correlates of disease. Hum Pathol. 2014;45(1):153-9. [CrossRef] [PubMed]

3. Sheff KW, York ER, Driebe EM, Barker BM, Rounsley SD, Waddell VG, BeckstromSternberg SM, Beckstrom-Sternberg JS, Keim PS, Engelthaler DM. Development of a rapid, cost-effective TaqMan Real-Time PCR Assay for identification and differentiation of Coccidioides immitis and Coccidioides posadasii. Med Mycol. 2010;48(3):466-9. [CrossRef] [PubMed] 